DOI: $10.2478 / \mathrm{v} \cdot 10169-012-0022-\mathrm{z}$

\title{
APPLICATION OF EXTENDED FINITE ELEMENT METHOD TO CRACKED CONCRETE ELEMENTS - NUMERICAL ASPECTS
}

\author{
J. BOBIŃSKI ${ }^{1}$, J. TEJCHMAN $^{1}$
}

\begin{abstract}
The paper deals with the application of the eXtended Finite Element Method (XFEM) to simulations of discrete macro-cracks in plain concrete specimens under tension, bending and shear. Fundamental relationships and basic discrete constitutive laws were described. The most important aspects of the numerical implementation were discussed. Advantages and disadvantages of the method were outlined.
\end{abstract}

Key words: concrete, crack, eXtended Finite Element Method (XFEM), implementation.

\section{INTRODUCTION}

Fracture is a fundamental phenomenon in quasi-brittle materials and its description requires the use of special enriched mathematical techniques. During a fracture process, micro-cracks first arise which change gradually into dominant distinct macroscopic discrete cracks up to rupture (Bažant and Planas [1], Lillu and van Mier [2]). Thus, a fracture process is generally subdivided into 2 main stages: appearance of narrow regions of intense deformation (including micro-cracks) and occurrence of macro-cracks (Tejchman and Bobiński [3]). The first stage (strain localization) can be numerically captured by continuous approaches based on elasto-plastic, damage mechanics, coupled elastic-plastic-damage and microplane constitutive laws enhanced by a characteristic length of microstructure [3]. In turn, macro-cracks (second stage) can be captured as a jump in a continuum displacement field by means of discontinuous methods including e.g. cohesive elements (interfaces) defined along finite element edges (ORTIZ and Pandolfi [4], Galvez et al. [5], Zhou and Molinari [6]) or strong discontinuities using elemental or nodal enrichments wherein cracks can arbitrarily propagate through finite elements (Belytschko et al. [7], Jirasek [8], Belytschko et al. [9], Simone and Sluys [10], OLIVER et al. [11]), which offer more flexibility for the crack path than interface elements. Usually, to describe the fracture behaviour of concrete, one approach is only used.

\footnotetext{
${ }^{1}$ Gdańsk University of Technology Civil Engineering and Environmental Engineering, Gdańsk-Wrzeszcz, Poland. e-mail: bobin@pg.gda.pl, tejchmk@pg.gda.pl
} 
The intention of the paper is to analyse some essential numerical aspects of the Extended Finite Element Method (XFEM). The method is based on the Partition of Unity concept (MELENK and BABUŠKA [12]) allowing for adding locally extra terms to the standard FE displacement field approximation for a better capture of displacement discontinuities. These extra terms are defined on a basis on a known analytical solution of the problem. Only selected nodes are enriched with additional terms. Thus, there is no need to modify the original FE mesh. The influence of different element types and different integration schemes in a cracked element definition was investigated during three different benchmark problems for concrete. Three different crack models and two different conditions for the crack propagation were analysed. In addition, the effect of some material parameters was studied.

Belytschko and Black [13] used XFEM for the first time to model cracks in elasto-brittle materials. To describe the stress field around the crack tip, asymptotic branch functions were proposed. Mö̈s and BеLYтsснко [14] formulated the model with branch functions for elements with crack tip and Heaviside jump function for elements cut entirely by a crack. Later XFEM was extended to deal with branching and intersecting cracks (Daux et al. [15]) and to simulate three-dimensional problems (Sukumar et al. [16]). XFEM was also used to analyze problems with weak discontinuities (like material interfaces), in fluid mechanics, modeling voids and holes, phase transformations, biofilms and dislocation problems as well. Wells and SLuYs [17] were the first to couple XFEM with cohesive cracks. Only Heaviside jump function was defined to describe the displacement jump across the discontinuity. MoËs and BelYtschкo [18] extended their model [14] by the Heaviside jump and branch functions to simulate cohesive cracks. ZI and Bецүтsснко [19] formulated a new crack tip element using linear ramp functions for the description of the crack tip location. Mergheim et al. [20] adopted the idea of HANSBo and HANSBo [21]. In this formulation there are no extra degrees of freedom in nodes. Instead of that any element with a crack is described by two overlapping standard finite elements with zero shape functions either of the left or on the right side of the discontinuity. Only displacement degrees of freedom are used, but extra phantom nodes have to be added in cracked elements to double standard nodes at the moment of cracking. This approach was later used by Song et al. [22] to simulate cohesive shear zones and by RABCZUK et al. [23] to handle crack tips inside elements.

The innovative points of our calculations are the most important numerical aspects of the XFEM implementation during three different boundary value problems (uniaxial tension, three point bending and mixed tension-shear) dealing with cracked concrete specimens. In the paper, different local crack propagation criteria and integration schemes after introducing a discontinuity were examined. The choice of a cohesive constitutive law and material parameters in softening was investigated. In addition, an implementation description of XFEM into the FE package ABAQUS was given.

The paper is organised as follows. Section 2 presents basic concepts and fundamental equations of XFEM. Continuum and discontinuous constitutive laws are shortly 
described in Section 3. Selected numerical aspects of the implementation are discussed in Section 4. Sections 5-7 presents numerical results of simulated benchmarks: uniaxial tension, three point bending and mixed tension-shear test. Finally, Section 8 includes conclusions and future plans.

\section{Theoretical Background of XFEM}

\subsection{Displacement FIELD}

The displacement field in the body $\Omega$ crossed by a discontinuity $\Gamma_{u}$ (Fig. 1) can be defined as (Belytschko and Black [13], Wells and Sluys [17])

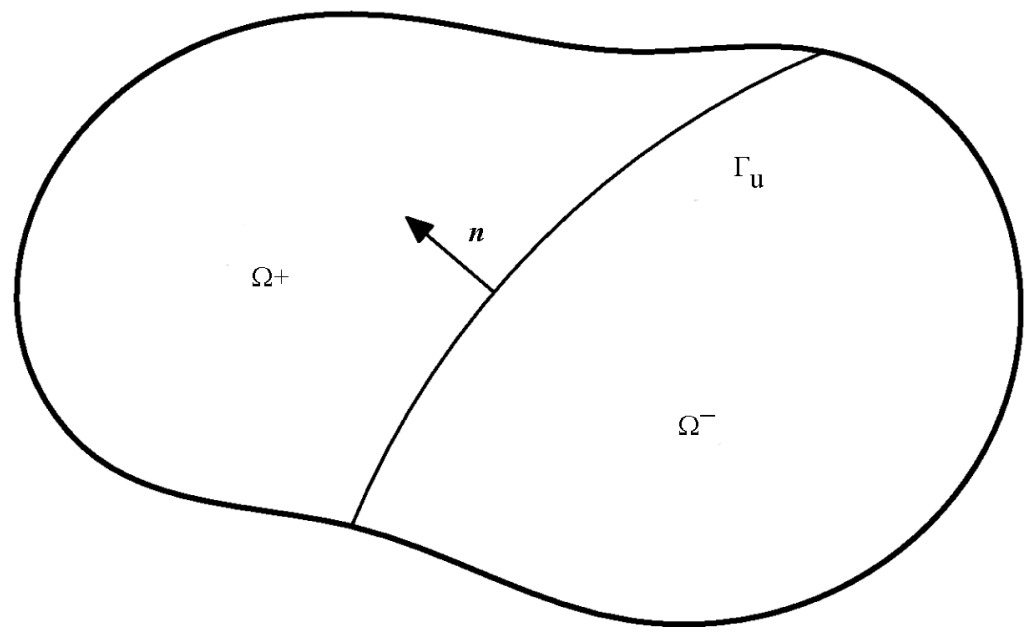

Fig. 1. Body crossed by discontinuity.

$$
\boldsymbol{u}(\boldsymbol{x}, t)=\hat{\boldsymbol{u}}(\boldsymbol{x}, t)+\Psi(\boldsymbol{x}) \tilde{\boldsymbol{u}}(\boldsymbol{x}, t),
$$

with the continuous functions $\hat{\boldsymbol{u}}$ and $\tilde{\boldsymbol{u}}$ and generalised step function $\Psi$

$$
\Psi(x)=\left\{\begin{array}{cc}
1 & x \in \Omega^{+} \\
-1 & x \in \Omega^{-}
\end{array},\right.
$$

In a finite element format, Eq. 1 can be written in general as

$$
\boldsymbol{u}(\boldsymbol{x})=N(\boldsymbol{x}) \boldsymbol{a}+\Psi(\boldsymbol{x}) \boldsymbol{N}(\boldsymbol{x}) \boldsymbol{b},
$$

where $\boldsymbol{N}$ contains the shape functions, $\boldsymbol{a}$ - the standard displacements at nodes and $\boldsymbol{b}$ - the enriched displacements (jumps) at nodes. Nodes belonging to cracked elements 
are solely enriched. In the paper, the alternative formulation called the shifted-basis enrichment was used (ZI and BeLYTschкo [19])

$$
u(x)=N(x) a+\left(\Psi(x)-\Psi\left(x_{I}\right)\right) N(x) b,
$$

with the diagonal matrices $\boldsymbol{\Psi}(\boldsymbol{x})$ and $\boldsymbol{\Psi}\left(\boldsymbol{x}_{\boldsymbol{I}}\right)$, respectively $\left(\boldsymbol{x}_{\boldsymbol{I}}\right.$ is the position of the node $I$ ). This formulation has two main advantages over the standard version (Eq. 2.3): the total nodal displacements are equal to the standard displacements $\boldsymbol{a}$, and the implementation of finite elements is simpler since two types of elements exist only.

\subsection{Discrete Weak equations}

Te discrete weak equations are defined as

$$
\begin{gathered}
\int_{\Omega} \boldsymbol{B}^{T} \boldsymbol{\sigma} \mathrm{d} \Omega=\int_{\Gamma_{u}} \boldsymbol{N}^{T} \overline{\boldsymbol{t}} \mathrm{d} \Gamma \\
\int_{\Omega}\left(\boldsymbol{\Psi}-\boldsymbol{\Psi}_{\boldsymbol{I}}\right) \boldsymbol{B}^{T} \boldsymbol{\sigma} \mathrm{d} \Omega+2 \int_{\Gamma_{d}} \boldsymbol{N}^{T} \boldsymbol{t} \mathrm{d} \Gamma=\int_{\Gamma_{u}}\left(\boldsymbol{\Psi}-\boldsymbol{\Psi}_{\boldsymbol{I}}\right) \boldsymbol{N}^{T} \overline{\mathrm{t}} \mathrm{d} \Gamma
\end{gathered}
$$

with the tractions $\overline{\boldsymbol{t}}$ applied on the boundary $\Gamma_{u}$, traction forces $\boldsymbol{t}$ acting on the discontinuity and the strain-nodal displacement matrix $\boldsymbol{B}$. The linearized equations of the total system are

$$
\left[\begin{array}{ll}
\boldsymbol{K}_{a a} & \boldsymbol{K}_{a b} \\
\boldsymbol{K}_{b a} & \boldsymbol{K}_{b b}
\end{array}\right]\left[\begin{array}{c}
\mathrm{d} \boldsymbol{a} \\
\mathrm{d} \boldsymbol{b}
\end{array}\right]=\left[\begin{array}{l}
\boldsymbol{f}_{a}^{e x t} \\
\boldsymbol{f}_{b}^{e x t}
\end{array}\right]-\left[\begin{array}{l}
\boldsymbol{f}_{a}^{i n t} \\
\boldsymbol{f}_{b}^{i n t}
\end{array}\right]
$$

with the blocks of the global stiffness matrix $\boldsymbol{K}$ defined as

$$
\begin{array}{cc}
\boldsymbol{K}_{a a}=\int_{\Omega} \boldsymbol{B}^{T} \boldsymbol{D} \boldsymbol{B} \mathrm{d} \Omega & \boldsymbol{K}_{a b}=\int_{\Omega} \boldsymbol{B}^{T} \boldsymbol{D} \boldsymbol{B}\left(\boldsymbol{\Psi}-\boldsymbol{\Psi}_{I}\right) \mathrm{d} \Omega \\
\boldsymbol{K}_{b a}=\int_{\Omega}\left(\boldsymbol{\Psi}-\boldsymbol{\Psi}_{I}\right) \boldsymbol{B}^{T} \boldsymbol{D} \boldsymbol{B} \mathrm{d} \Omega & \boldsymbol{K}_{b b}=\int_{\Omega}\left(\boldsymbol{\Psi}-\boldsymbol{\Psi}_{I}\right) \boldsymbol{B}^{T} \boldsymbol{D} \boldsymbol{B}\left(\boldsymbol{\Psi}-\boldsymbol{\Psi}_{I}\right) \mathrm{d} \Omega+4 \int_{\Gamma_{d}} \boldsymbol{N}^{T} \boldsymbol{T} \boldsymbol{N} \mathrm{d} \Gamma
\end{array},
$$

where $\boldsymbol{T}$ is the stiffness matrix at the discontinuity, and the force vectors are equal to

$$
\begin{array}{cc}
\boldsymbol{f}_{a}^{\text {ext }}=\int_{\Gamma_{u}} N^{T} \bar{t} \mathrm{~d} \Gamma & \boldsymbol{f}_{a}^{\text {int }}=\int_{\Omega} B^{T} \sigma \mathrm{d} \Omega \\
\boldsymbol{f}_{b}^{\text {ext }}=\int_{\Gamma_{u}}\left(\boldsymbol{\Psi}-\boldsymbol{\Psi}_{I}\right) N^{T} \overline{\boldsymbol{t}} \mathrm{d} \Gamma & \boldsymbol{f}_{b}^{\text {int }}=\int_{\Omega}\left(\boldsymbol{\Psi}-\boldsymbol{\Psi}_{I}\right) \boldsymbol{B}^{T} \sigma \mathrm{d} \Omega+2 \int_{\Gamma_{d}} N^{T} \boldsymbol{t} \mathrm{d} \Gamma
\end{array}
$$


The strain rate in the bulk continuum can be calculated as

$$
\dot{\varepsilon}=\boldsymbol{B} \dot{a}+\left(\Psi-\Psi_{I}\right) B \dot{b}
$$

whereas the rate of the displacement jump $[[\boldsymbol{u}]]$ at the discontinuity is

$$
[[\dot{u}]]=2 N \dot{b} .
$$

\section{Constitutive Relationship}

In an uncracked region, a linear elastic constitutive law between stresses $\sigma$ and strains $\varepsilon$ was always assumed

$$
\sigma=D^{e}: \varepsilon,
$$

where $\boldsymbol{D}^{e}$ is the linear elastic material matrix.

To activate a crack, the Rankine condition was fulfilled at least in one integration point of the finite element at the front of the crack tip

$$
\max \left\{\sigma_{1}, \sigma_{2}, \sigma_{3}\right\}>f_{t},
$$

where $\sigma_{1}, \sigma_{2}, \sigma_{3}$ are the principal stresses and $f_{t}$ is the tensile strength. This inequality can be also verified at the crack tip (Mariani and Perego [24]).

To find a direction of the crack propagation, the direction of the crack extension was assumed to be perpendicular to the direction of the maximum principal stress. To smoothen the stress field around the crack tip, the average stresses $\sigma^{*}$ was used for determining the crack direction according to WeLLs and SLuYs [17]

$$
\sigma^{*}=\int_{V} \sigma w \mathrm{~d} V,
$$

where the domain $V$ is the semicircle at the front of the crack tip and the weight function $w$ was defined as

$$
w=\frac{1}{(2 \pi)^{3 / 2} l^{3}} \exp \left(-\frac{r^{2}}{2 l^{2}}\right) .
$$

Herein $l$ is the averaging length (usually equal to 3 times the average element size) and $r$ denotes the distance between the integration point and crack tip (note that this operation does not intend to introduce a length of microstructure into the model). Other criteria may be also formulated. Mariani and Perego [24] used higher order polynomials for a better description of the stress (and also the displacement) state around the crack tip. The stresses in the crack tip were determined using interpolation from 
nodal values. OLIVER et al. [25] formulated so-called global tracking algorithm. The propagation directions of all cracks were determined globally by solving a stationary anisotropic heat conduction problem. MöEs and BeLYTschкo [18] assumed that cohesive tractions had no influence on the crack propagation direction and used the maximum circumferential stress criterion from Linear Elastic Fracture Mechanics (LEFM). In order to describe crack displacements, we assumed the following format of the loading function within a discrete cohesive law following WeLls and SLuYs [17]

$$
f\left(\left[\left[u_{n}\right]\right], \kappa\right)=\left[\left[u_{n}\right]\right]-\kappa,
$$

with the history parameter $\kappa$ equal to the maximum value of the displacement jump $\left[\left[u_{n}\right]\right]$ achieved during loading. The softening of the normal component of traction vector was described using either an exponential relationship

$$
t_{n}=f_{t} \exp \left(-\frac{f_{t} \kappa}{G_{f}}\right)
$$

or a linear one

$$
t_{n}=f_{t}\left(1-\frac{\kappa}{\kappa_{u}}\right), \quad \kappa_{u}=\frac{2 G_{f}}{f_{t}},
$$

where $G_{f}$ is the fracture energy. During unloading, the secant stiffness was used with a return to the origin (damage format). In a compressive regime, the penalty stiffness in the normal direction $T_{N}$ was assumed. In the tangent direction, a linear relationship between a displacement jump and traction was defined with the stiffness $T_{S}$. Since a transition between tension and compression and inversely in a normal direction in the approach by WelLS and SLuYs [17] may cause sudden changes in the material stiffness contributing to convergence problems, to overcome these problems we modified a linear softening curve in a normal direction according to Cox [26] in some simulation cases

$$
t_{n}=f_{t}\left(1-\frac{\left[\left[u_{n}\right]\right]}{\kappa_{u}}\right)\left(1-\exp \left(-d_{f} \frac{\left[\left[u_{n}\right]\right]}{\kappa_{u}}\right)\right),
$$

where $d_{f}$ is the drop factor. With increasing the value of $d_{f}$, the influence of the second term diminishes.

Discrete cohesive laws may also be based on an effective displacement formulation. Mariani and Perego [24] were the first who used this approach in the context of XFEM. In the paper, a discrete constitutive law proposed by UNGER et al. [27] was also taken advantage of. This formulation followed a standard discrete law based on effective displacements

$$
\left[\left[u_{e f f}\right]\right]=\sqrt{\left\langle\left[\left[u_{n}\right]\right]\right\rangle^{2}+\beta^{2}\left[\left[u_{s}\right]\right]^{2}},
$$


wherein the coefficient $\beta$ takes into account the coupling between the failure mode I and failure mode II. The loading function was defined as

$$
f\left(\left[\left[u_{e f f}\right]\right], \kappa\right)=\left[\left[u_{e f f}\right]\right]-\kappa
$$

with the history parameter $\kappa$ equal to the maximum value of the effective displacement $\left[\left[u_{e f f}\right]\right]$ obtained during loading. The normal and shear tractions were evaluated as

$$
t_{n}=\frac{t_{e f f}}{\left[\left[u_{e f f}\right]\right]}\left[\left[u_{n}\right]\right], \quad t_{s}=\beta^{2} \frac{t_{e f f}}{\left[\left[u_{e f f}\right]\right]}\left[\left[u_{s}\right]\right] .
$$

This constitutive law assumed also unloading to the origin. A softening function was defined as

$$
t_{\text {eff }}=\left\{\begin{array}{cc}
K_{p} \kappa & \kappa<\kappa_{0} \\
f_{t} \exp \left(-f_{t}\left(\kappa-\kappa_{0}\right) / G_{f}\right) & \kappa \geq \kappa_{0}
\end{array}, \quad \kappa_{0}=\frac{f_{t}}{K_{p}},\right.
$$

which includes a linear regime before the peak $\left(K_{p}\right.$ is the penalty stiffness).

\section{IMPLEMENTATION}

The inclusion of enriched displacement jumps $\boldsymbol{b}$ requires several modifications of standard FE codes. The final number of extra degrees of freedom $\boldsymbol{b}$ is unknown at the beginning and it may grow during calculations. Therefore special techniques are required to handle the extra data. If an essential boundary condition is specified at a node with enriched degrees of freedom, the additional condition $b=0$ has to be added at this node. A new crack segment can be defined at the converged configuration only. After defining a new segment, a current increment has to be restarted. The nodes that share the edge with a crack tip cannot be enriched to preserve the displacement field continuity. Standard XFEM algorithms (e.g. WeLls and SLuYs [17]) put some limitations on a crack definition. A crack consists of several straight segments. Crack vertices can be placed at element edges only (not in vertices and inside of elements) and adjacent segments have to share the same point (due to the crack continuity condition). To avoid defining crack vertices in element vertices and creating degenerated crack elements, the minimum distances $v_{\min }$ and $l_{\min }$ to the element vertex or the minimum distance $s_{\min }$ to the element sides, not touched by a crack segment, between points $P$ and $Q$ are defined (Fig. 2, superscript denotes the element side). The distance $v_{\min }$ was measured using the Euclidean norm, while the distance $l_{\min }$ was calculated along the element side (they were identical in elements with straight edges). We assumed the relative minimum distance to the element vertex equal to $1 \%$ of the side length.

The introduction of a discontinuity line in cracked element requires a new integration scheme for calculating the strains, stresses, internal force vector and stiffness matrix. In this paper the 3-integration schemes for 3-node triangles were chosen with: 
a)

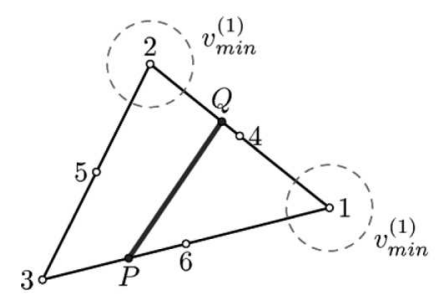

b)

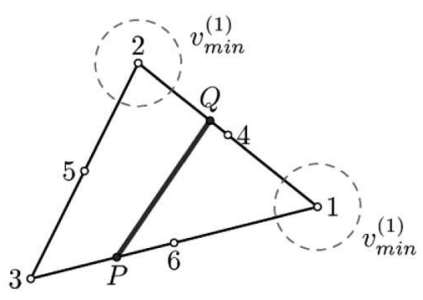

c)

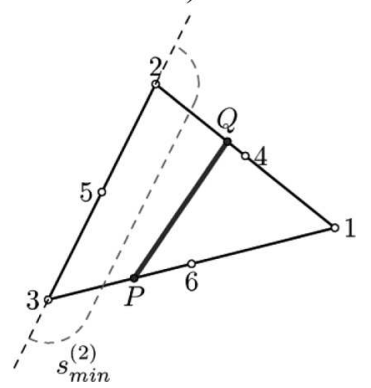

Fig. 2. Minimum distances between crack segments and triangle vertices/sides: a) $v_{\min }$, b) $l_{\min }$ and c) $s_{\min }$.

3 integration points (AsFerg et al. [28]), 7 integration points or 21 integration points (Wells and SluYs [17]) (Fig. 3). In quadrilaterals, totally 12 integration points were defined (DumstorfF [29]) (Fig. 4). In all calculation cases, two integration points on a discontinuity line were defined. XFEM was implemented into the commercial FE package ABAQUS/Standard. A finite element with a displacement jump was defined using the UEL (user element) subroutine. The displacement jumps were defined by activating rotational degrees of freedom in the model. One node user elements with no stiffness and zero force were defined at nodes with defined boundary conditions (to impose an additional condition on displacement jumps). The data about residuum forces and displacement corrections were gathered independently of ABAQUS (they were taken from user elements). It allowed for an independent control of the convergence process and to detect converged iterations. To drive the convergence process in ABAQUS and to enable extending cracks without starting a new increment, another user element was added. It had no stiffness, but it returned very large force vector in iterations when the convergence (start of the new increment) was not permitted. An extra module was written to visualise crack patterns.

a)

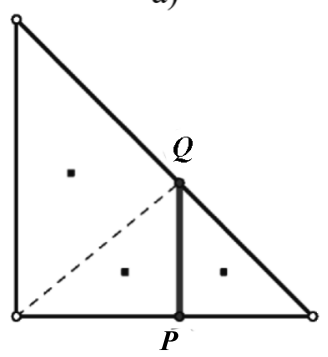

b)

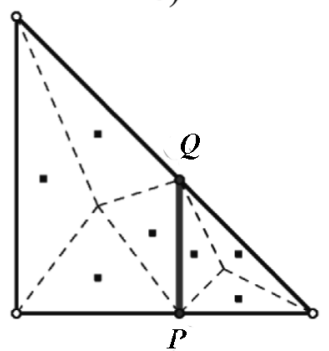

c)

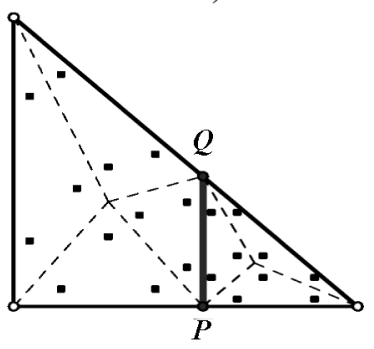

Fig. 3. Integration schemes in 3-node triangles: a) 3 integration points, b) 7 integration points and c) 21 integration points. 
a)

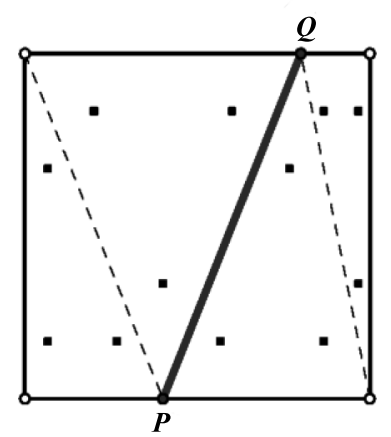

b)

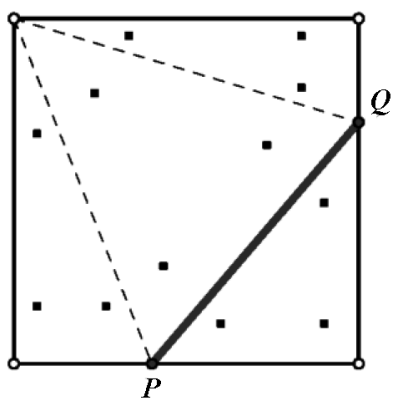

Fig. 4. Integration schemes in 4-node quadrilaterals: a) divided into 2 quadrilaterals and b) divided into trapezoid and triangle.

\section{UNIAXIAL TENSION OF CONCRETE}

First, a simple uniaxial tension test was simulated. The width of the specimen was $100 \mathrm{~mm}$, height $150 \mathrm{~mm}$ and thickness $1.0 \mathrm{~m}$ (Fig. 5). The thickness size did not influence the normalized FE results. All nodes along the bottom were fixed in the vertical direction. The vertical tensile deformation was imposed by enforcing the vertical dis-

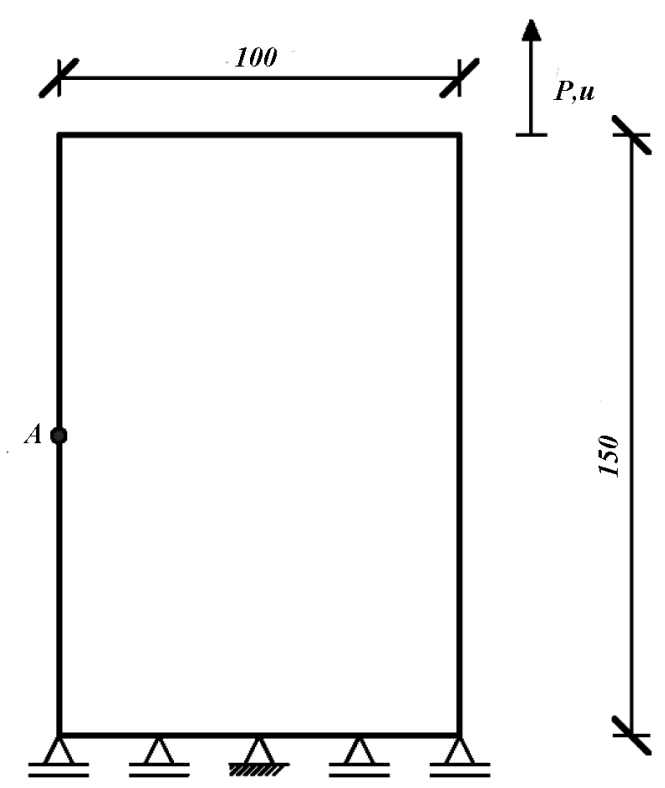

Fig. 5. Uniaxial tension numerical test: geometry and boundary conditions $(P$ - vertical force along upper edge, $u$ - vertical displacement of upper edge, $A$ - crack start point). 
placement increment of all nodes along the upper edge by the amount of $\Delta u=0.1 \mathrm{~mm}$. To preserve the stability of the specimen, the node in the middle of bottom was fixed in the horizontal direction. The starting point of the crack propagation was defined in the middle of the left edge. The modulus of elasticity was equal to $E=30 \mathrm{GPa}$, the Poisson's ratio was $v=0.2$, the tensile strength $f_{t}=3 \mathrm{MPa}$ and the fracture energy $G_{f}=100 \mathrm{~N} / \mathrm{m}$. Simple exponential softening was defined. The discrete cohesive law by WeLls and Sluys (2001) was adopted (Eqs.3.5-3.6). The penalty stiffness in a normal and tangential directions were equal to $T_{N}=10^{14} \mathrm{~N} / \mathrm{m}$ and $T_{S}=10^{12} \mathrm{~N} / \mathrm{m}$, respectively. The local stress averaging criterion was chosen (averaging length was taken as $l=0$ ).

\subsection{Mesh SIZE}

To examine the mesh insensitivity, simulations were performed with three different 3-node triangle FE-meshes: with the coarse (600 elements), medium (2400 elements) and fine one (5400 elements). The integration scheme by Asferg et al. [28] in cracked elements was assumed.

Almost the same force-displacement curves were obtained with a medium and fine mesh (Fig. 6). Small discrepancies with a coarse mesh were caused by the chosen algorithm of the crack propagation (see Section 5.2). A horizontal crack was also properly reproduced (Fig. 7).

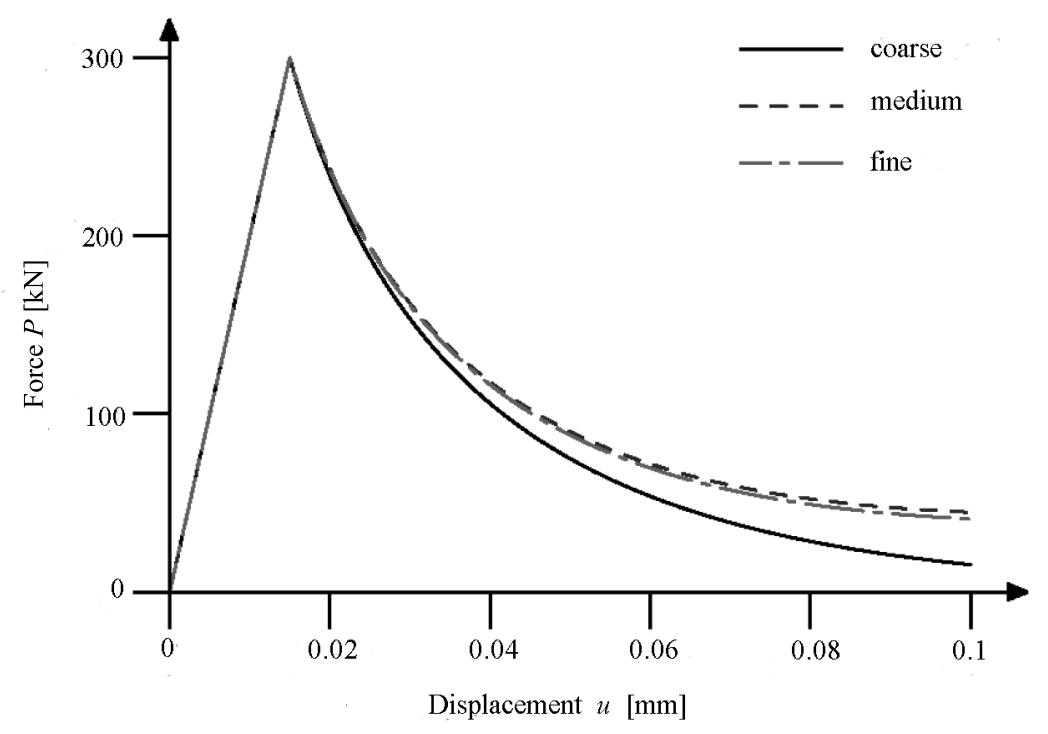

Fig. 6. Uniaxial tension numerical test: force-displacement diagrams for different FE meshes. 
a)

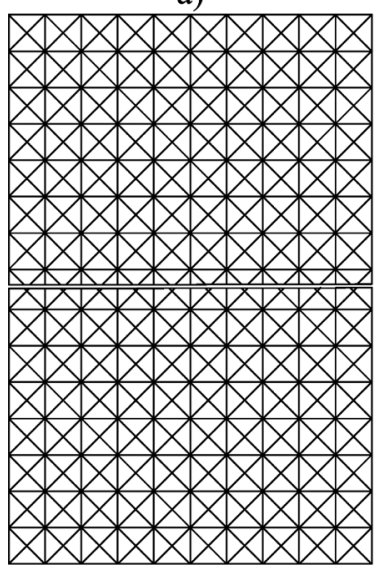

b)

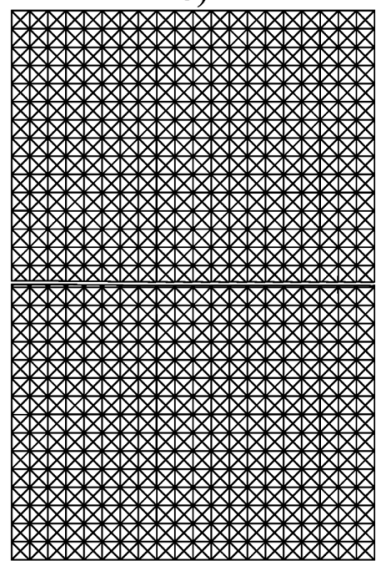

c)

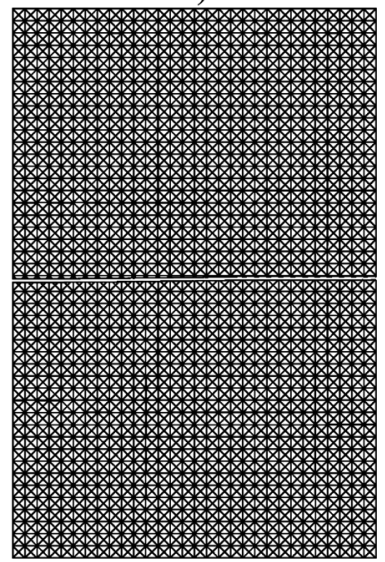

Fig. 7. Uniaxial tension numerical test: formation of horizontal crack for different meshes: a) coarse, b) medium and c) fine (displacements were magnified by scale factor of 10).

Next, the influence of the element type and integration scheme in cracked elements was investigated. Three-node cracked triangles with 3,7 or 21 integration points and 4-node quadrilateral cracked elements with 12 integration points were assumed. The obtained force-displacement curves for a medium mesh are presented in Fig. 8. They are similar as in Fig. 6. Therefore, in later simulations, the algorithm Asferg et al. [28] with 3 integration points was assumed for 3-node triangles.

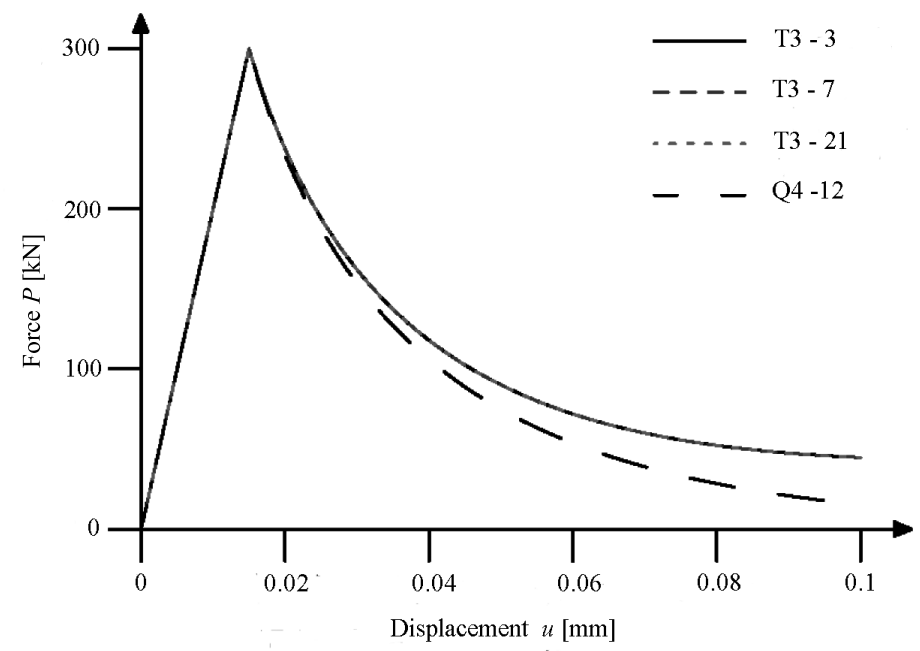

Fig. 8. Uniaxial tension numerical test: influence of element type and integration scheme on force-displacement diagram. 


\subsection{DireCtion OF CRACK PROPAGATION}

All simulations were performed with the aid of a condition for the principal stress direction. It means that the direction of the next crack segment was determined on the basis of the equilibrium configuration by taking into account earlier created crack segments. As a consequence, the intermediate stress field could be in general non-uniform and cause not-ideally horizontal crack (observed in simulations with triangular finite elements). Therefore the simulations with 3-node triangle meshes were repeated by assuming a fixed horizontal crack direction from the beginning (Fig. 9). The calculated force-displacement curves are the same for all meshes.

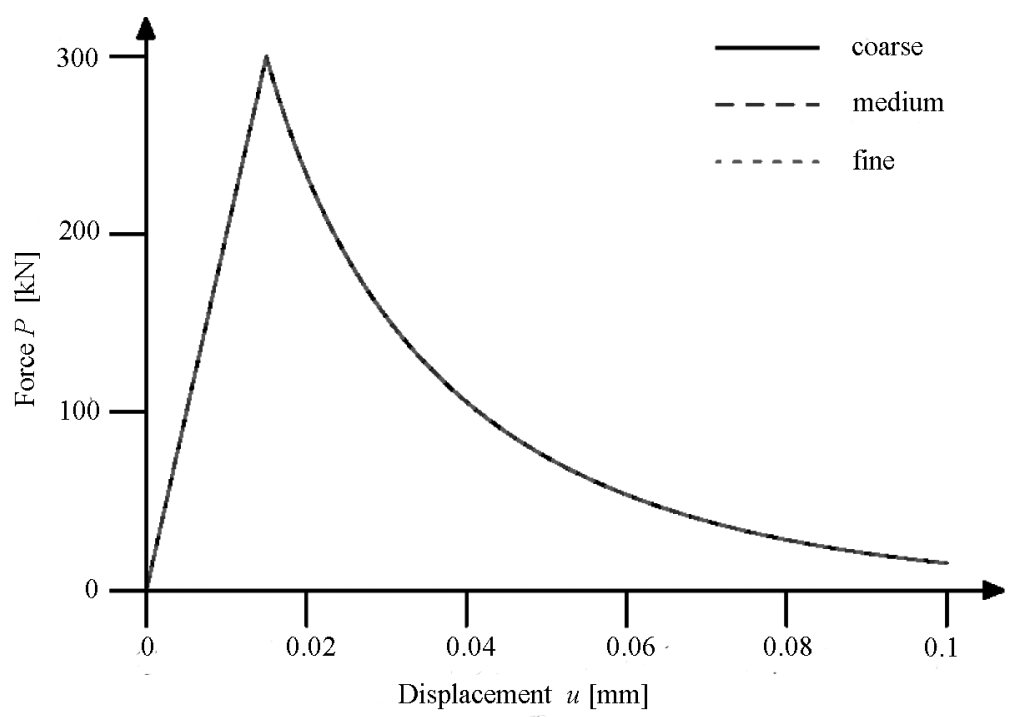

Fig. 9. Uniaxial tension numerical test: force-displacement diagrams for different FE meshes with fixed horizontal crack propagation direction.

The uniaxial tension test was also simulated using the discrete Cox law (Eq. 3.8). The influence of the drop factor $d_{f}$ on the force-displacement diagrams was examined (Fig. 10). A horizontal crack was assumed in advance. A medium mesh with 3-node triangles was used. With increasing factor $d_{f}$, the force-displacement curves approached the results of Fig. 6. In later simulations, the parameter $d_{f}=10^{4}$ was chosen.

\section{ThreE-POINT BENDING OF CONCRETE}

Next, the simulations of a three-point bending test of notched concrete beams were carried out. This experiment was performed by Le BELLEGo et al. [30] and later numerically simulated by Le Bellego at al. [30], Rodriguez-Ferran et al [31] and Skarżynski 


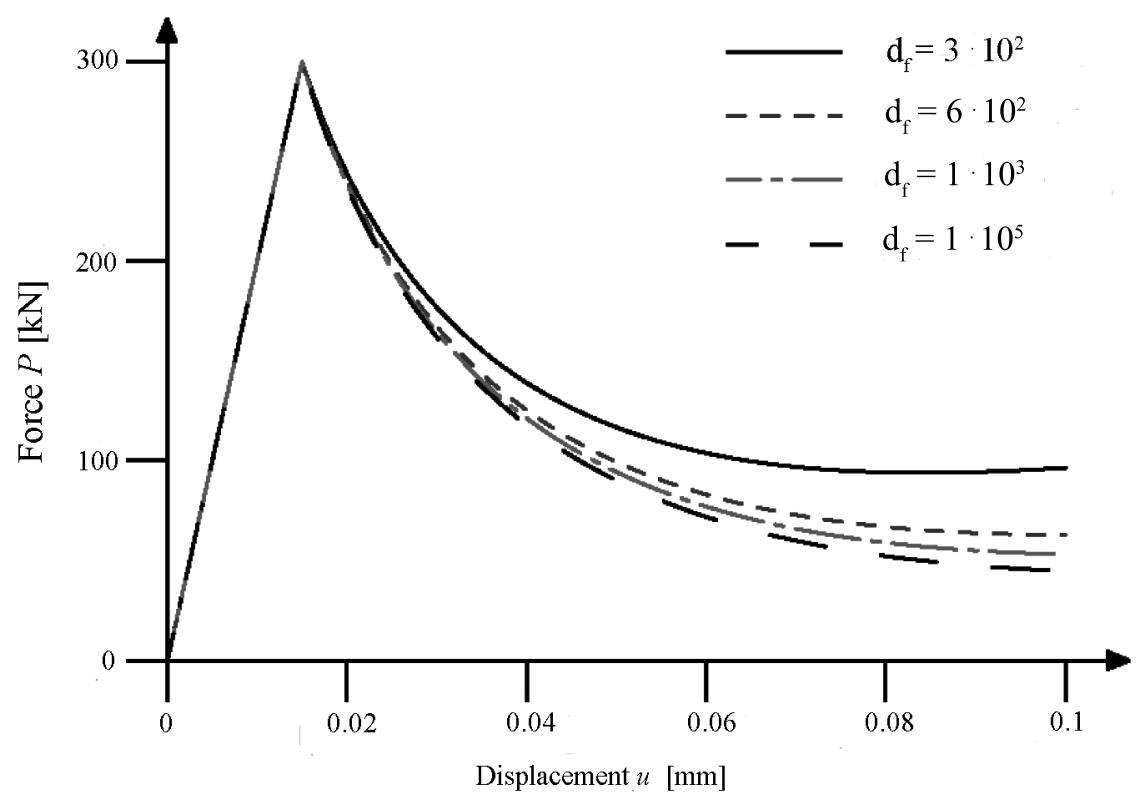

Fig. 10. Uniaxial tension numerical test: force-displacement diagrams for different drop factors $d_{f}$ from Eq. 3.8.

and Tejchman [32] with a damage approach with non-local softening. Three different beam sizes were numerically investigated: small $(h=8 \mathrm{~cm})$, medium $(h=16 \mathrm{~cm})$ and large one $(h=32 \mathrm{~cm})$. The span length of the beam was equal to $L=3 h$ (Fig. 11). The loading was prescribed at the top edge at the mid-span via the vertical displacement. In the simulations, the Young modulus $E=38.5 \mathrm{GPa}$ and Poisson ratio $v=0.2$ were taken. The tensile strength was equal to $f_{t}=3.2 \mathrm{MPa}$. The exponential softening with the fracture energy $G_{f}=80 \mathrm{~N} / \mathrm{m}$ was defined. The discrete law by Cox was chosen with the stiffness $T_{S}=0$. A fixed vertical crack direction above the notch was used. The stress averaging length was $l=1 \mathrm{~cm}$. The material parameters were the same for all beams without taking into account a stochastic distribution of concrete properties (due to a its heterogeneous structure) depending upon the beam size. Three different meshes with 3068, 4956 and 9132 3-node constant strain triangles were defined for a small, medium and large beam, respectively. The crack starting points were located at the left side near the node at the line of the symmetry of the each beam. Three integration points were defined in cracked elements.

Figure 12 shows the calculated force-displacement diagrams for notched concrete beams as compared with the experimental curves. Since it was not our intention to reproduce perfectly experiments, some differences can be seen, especially in a linear elastic regime. In turn, the softening behaviour of beams was properly captured. The maximum calculated force was equal to $2945 \mathrm{~N}, 5185 \mathrm{~N}$ and $8784 \mathrm{~N}$ for the small, 

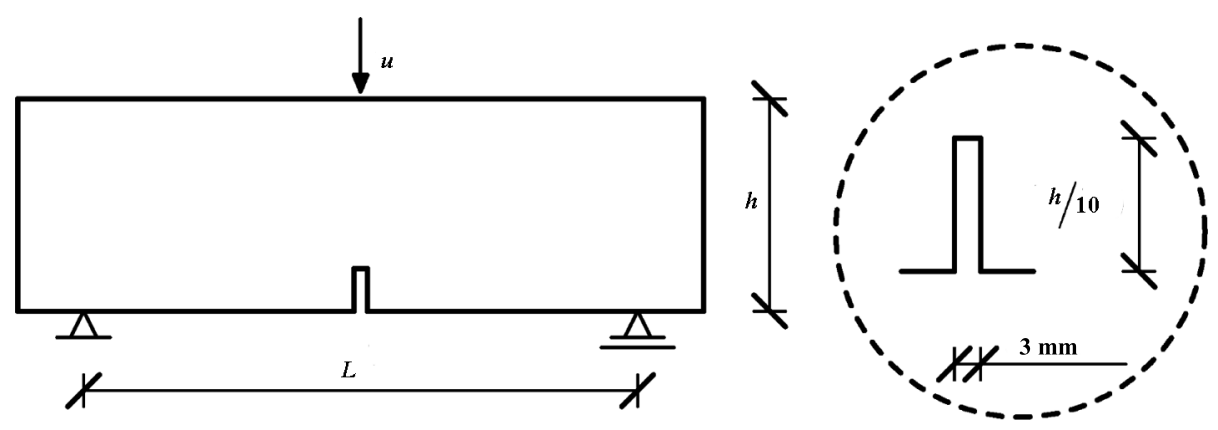

Fig. 11. Three-point bending test by Le Bellego et al. [30].

medium and large beam, respectively. It was similar as in experiments, thus a strong size effect in concrete beams (expressed by the increasing bearing load capacity with decreasing size) was also properly reproduced.

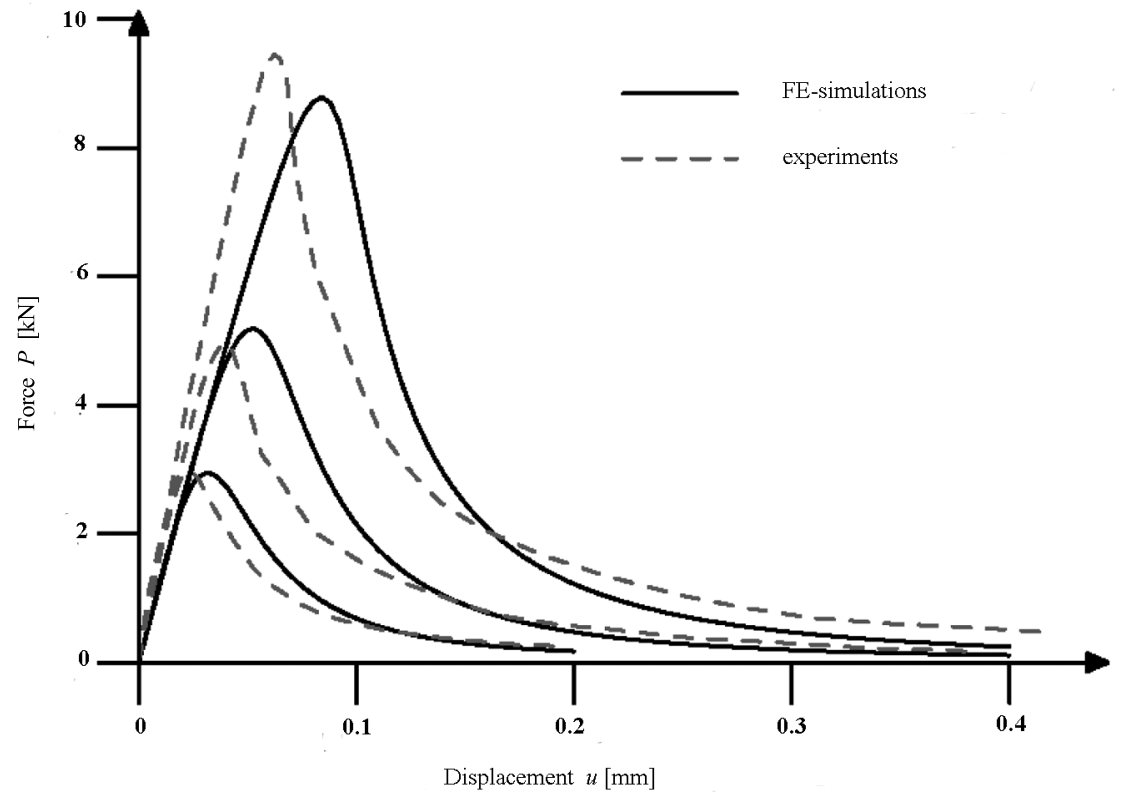

Fig. 12. Three-point bending test: experimental and calculated force-displacement diagrams for different beam sizes: large (upper curve), medium (mid-curve) and small (lower curve).

The obtained displaced mesh for a small notched concrete beam is presented in Fig. 13. Due to the assumed propagation direction, the calculated crack is obviously perfectly vertical. In turn, the use of a crack propagation direction based on the maximum principal stress resulted in a sudden change of the crack direction at the certain 
stage of the beam deflection. The reason was a biaxial tension stress state built at the front of the crack tip. The vertical tensile stresses became larger than the horizontal ones and the crack begun to turn by $90^{\circ}$ in the left (or right) direction. This problem merits further investigations.

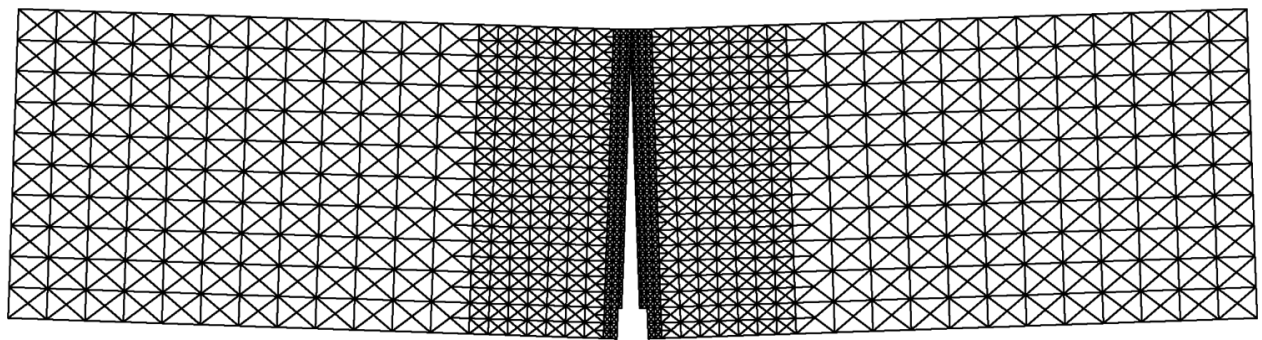

Fig. 13. Three-point bending test: numerical vertical crack in small-size notched concrete beam.

\section{Mixed TENSION-SHEAR OF CONCRETE}

A double-edge notched specimen (DEN) (Fig. 14) under different loading paths of combined shear and tension was analysed (so-called the Nooru-Moнamed [33] benchmark

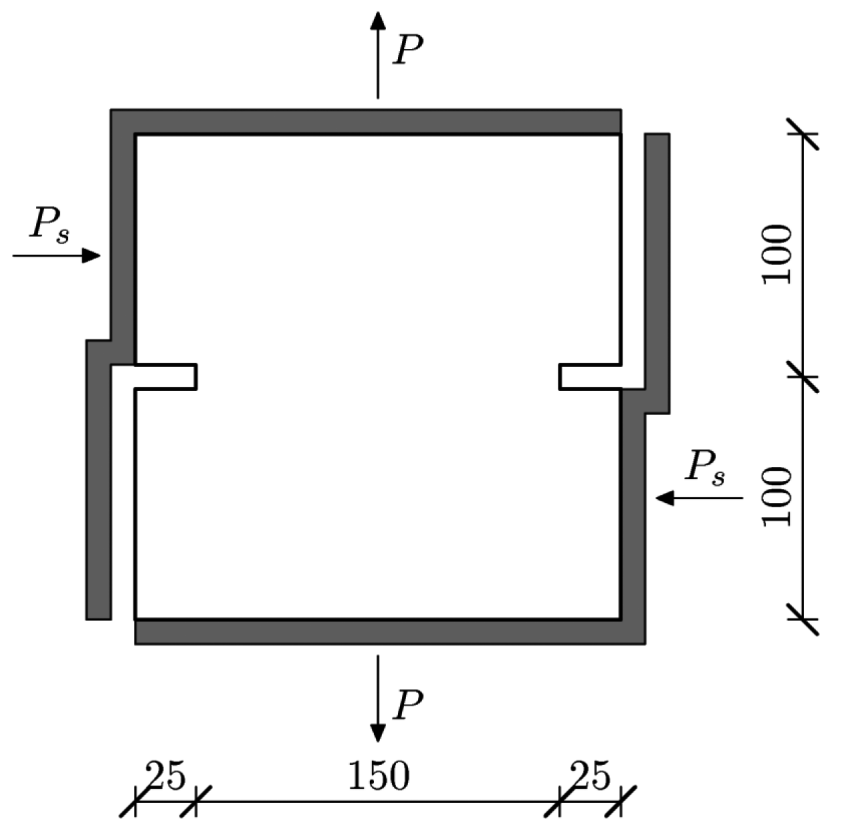

Fig. 14. Nooru-Mohamed [33] test: geometry and boundary conditions. 
problem). The width of the specimen was $50 \mathrm{~mm}$. First, a shear force $P_{s}$ was applied until it reached a specific value, while the horizontal edges were free. At the second stage, the shear force remained constant and the vertical tensile displacement was prescribed. Two curved cracks with an inclination depending on the value of the shear force were experimentally obtained, i.e. almost horizontal for small values of $P_{s}$ and highly curved for large values of $P_{s}$ (Fig. 15).

a)

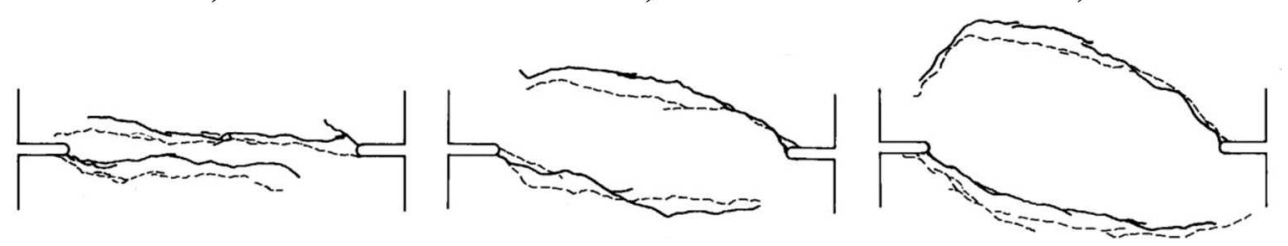

Fig. 15. Nooru-Mohamed [33] experimental cracks for different shear force: a) $\left.P_{s}=5 \mathrm{kN}, \mathrm{b}\right) P_{s}=10 \mathrm{kN}$ and c) $P_{s}=15 \mathrm{kN}$.

The following constants were assumed in elasticity for simulations: the Young modulus $E=38.2 \mathrm{GPa}$ and the Poisson's ratio $v=0.2$. The tensile strength was equal to $f_{t}=3 \mathrm{MPa}$. The exponential softening with the fracture energy $G_{f}=100 \mathrm{~N} / \mathrm{m}$ were assumed for cohesive cracks. Two crack starting points were defined near the notch corners. To calculate the crack propagation direction the criterion based on a direction of the maximum principal stress was used. The stress averaging length was $l=1 \mathrm{~cm}$.

\subsection{Mesh insensitivity}

Two meshes were used: the first mesh consisted of 3840 3-node triangle elements and the second one of 10604 -node quad elements. Figure 16 shows the calculated cracks obtained with two different meshes for the shear force $P_{s}=5 \mathrm{kN}$ (path ' $4 \mathrm{a}$ ') with the Cox discrete law (Eq. 3.8) and $T_{S}=0$. In both cases, inclined cracks were obtained (slightly too curved as compared to experiment). The force-displacement diagrams were similar (Fig. 17). The differences with the experimental curve were caused by the over-predicted experimental tensile strength.

The simulations for the shear force $P_{s}=10 \mathrm{kN}$ were performed using the Unger discrete model with the parameter $\beta=0$ (Eqs. 3.9-3.12). The penalty stiffness was $K_{p}=10^{13} \mathrm{~N} / \mathrm{m}$. For two different meshes, again too slightly curved cracks were calculated (Fig. 18). The shape of the obtained force-displacement curves was similar (Fig. 19), however the curves were again located above the experimental ones.

The calculated cracks and force-displacement curves are presented in Fig. 20 with the different coupling parameter $\beta\left(P_{s}=5 \mathrm{kN}\right)$. The results indicate rather surprisingly that the effect of $\beta$ is negligible. The penalty stiffness was $K_{p}=10^{12} \mathrm{~N} / \mathrm{m}$. 
a)

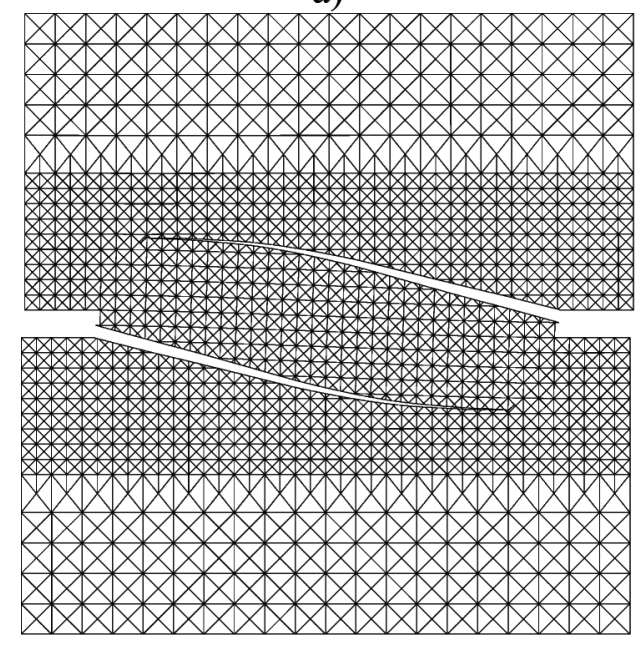

b)

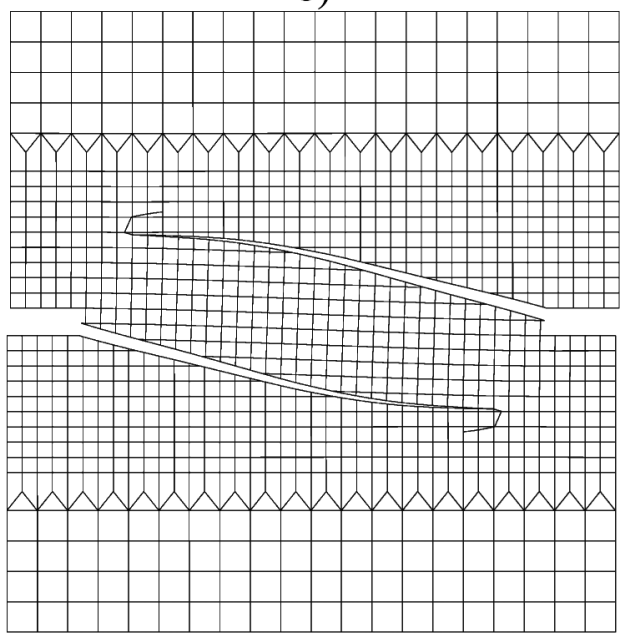

Fig. 16. Nooru-Mohamed [33] test: calculated cracks at $P_{s}=5 \mathrm{kN}$ for two different finite element types: a) 3-node triangles and b) 4-node quads (with crack model by Cox [26]).

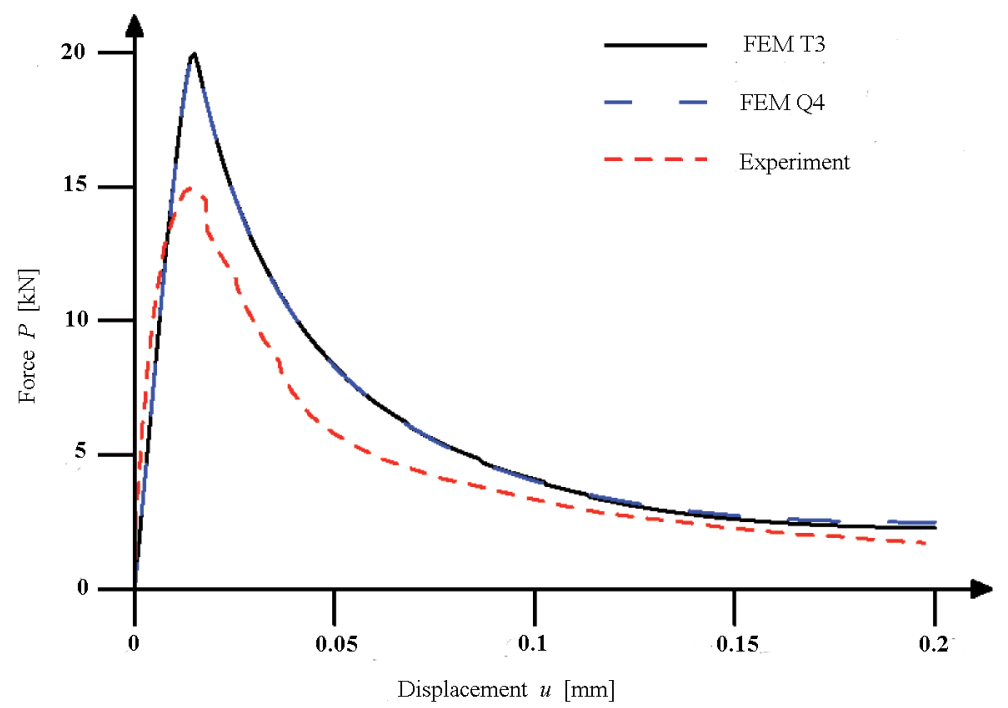

Fig. 17. Nooru-Mohamed [33] test: calculated force-displacement curves for 2 different FE element types at $P_{s}=5 \mathrm{kN}$ compared with experiment (using model by Cox [26]). 
a)

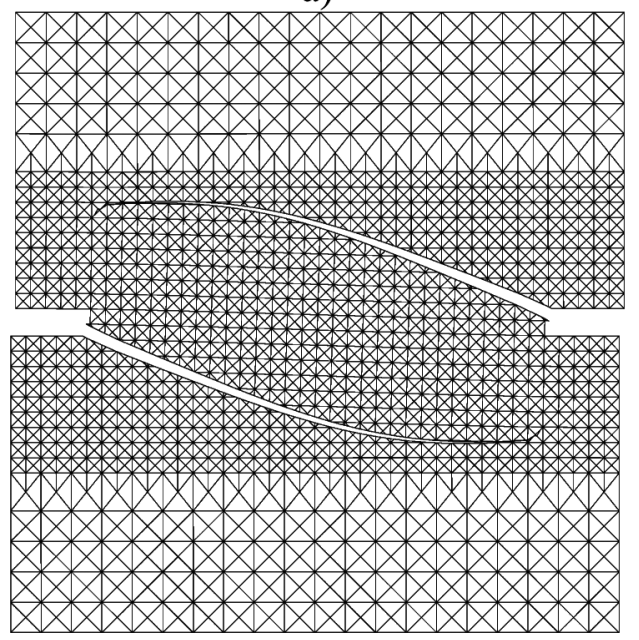

b)

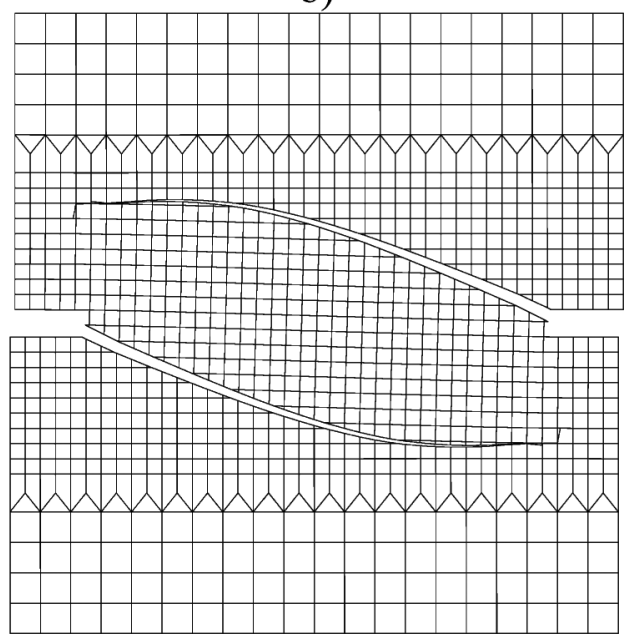

Fig. 18. Nooru-Mohamed problem: calculated cracks for different FE element types at $P_{s}=10 \mathrm{kN}$ : a) 3-node triangles and b) 4-node quads (with crack model by Unger et al. [27]).

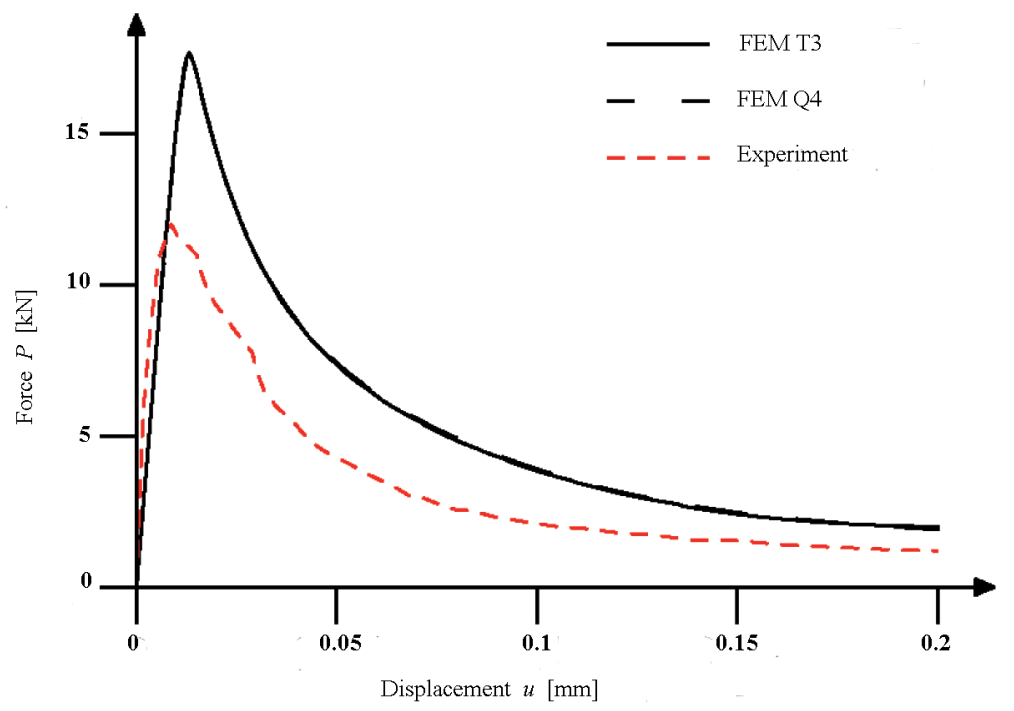

Fig. 19. Nooru-Mohamed [33] test: calculated force-displacement curves for different FE element types at $P_{s}=10 \mathrm{kN}$ compared with experiment (with crack model by Unger et al. [27]). 
a)

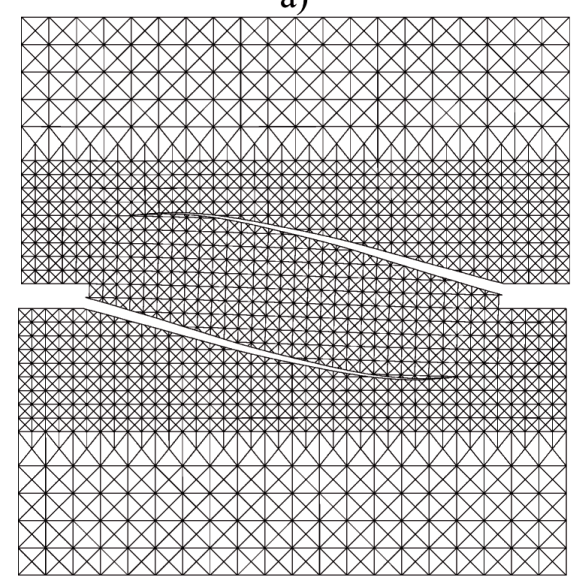

b)

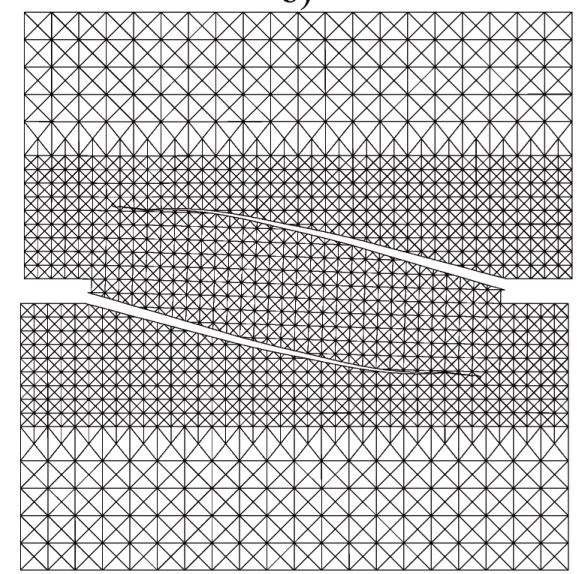

c)

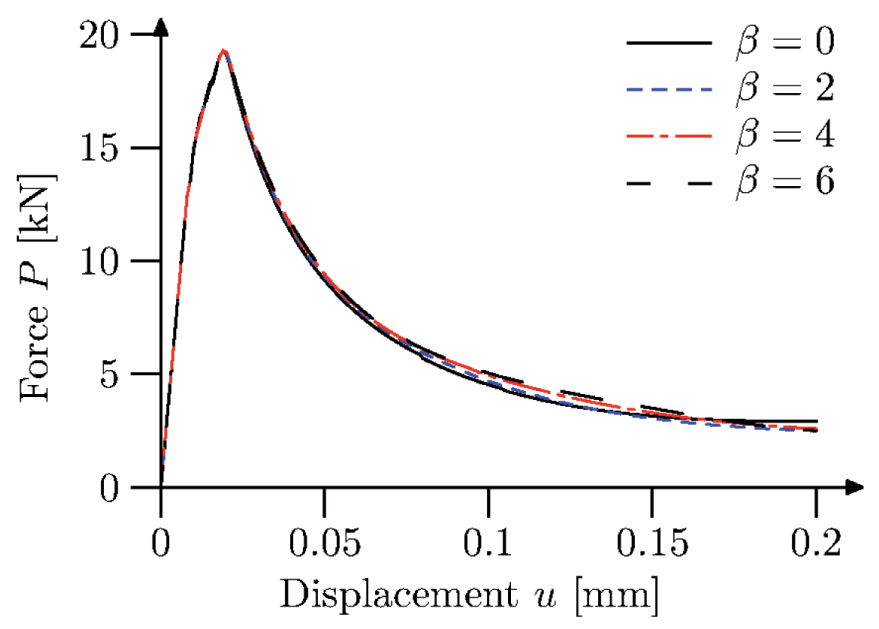

Fig. 20. Nooru-Mohamed [33] test: calculated cracks with coupling parameter $\beta$ (a) $\beta=0$ and b) $\beta=4$ ) and force-displacement curves c) (model by Unger et al. [27]).

The effect of the averaging length $l$ (Eq. 3.4) is shown in Fig. 21. The Cox discrete law was used again. The averaging length does not affect the force-displacement diagram, but the crack direction.

Finally, FE simulations were performed with the different minimum distance from elements' vertices. A relative minimum distance was taken as $1 \%$ (the default value in remaining simulations), $5 \%, 10 \%$ or $20 \%$ of the side length. Similar crack patterns and force-displacement diagrams are obtained, however cracks for larger values are less smooth (Fig. 22). 
a)

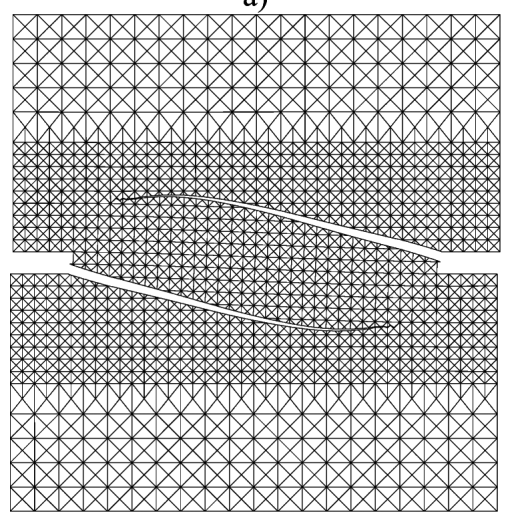

b)
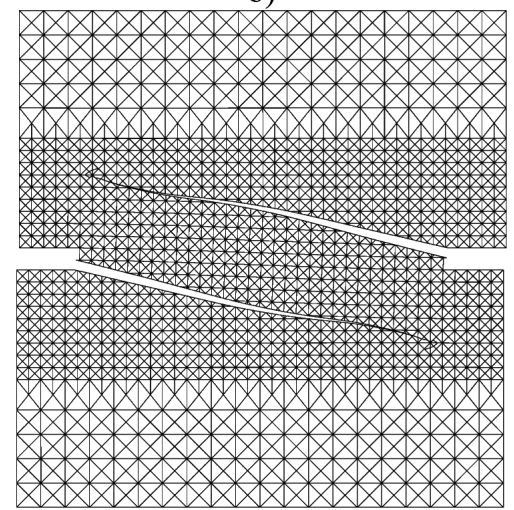

Fig. 21. Nooru-Mohamed [33] test: calculated cracks for different averaging length $l$ : (Eq. 3.4): a) $l=0.5 \mathrm{~mm}$ and b) $l=2 \mathrm{~mm}$ (crack model by Cox [26]).

a)

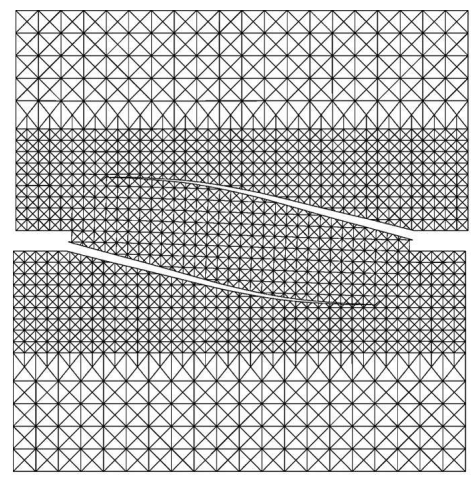

c)
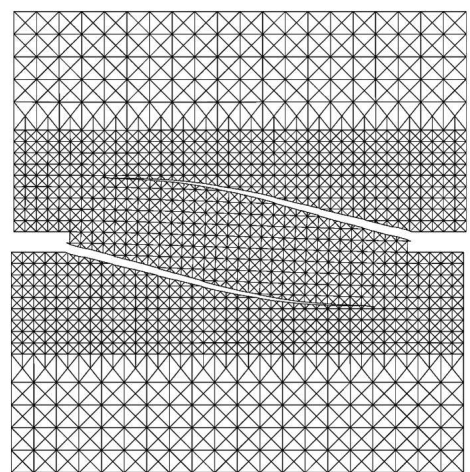

b)
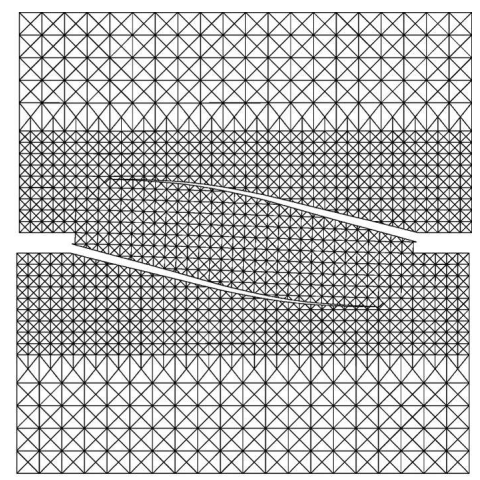

d)

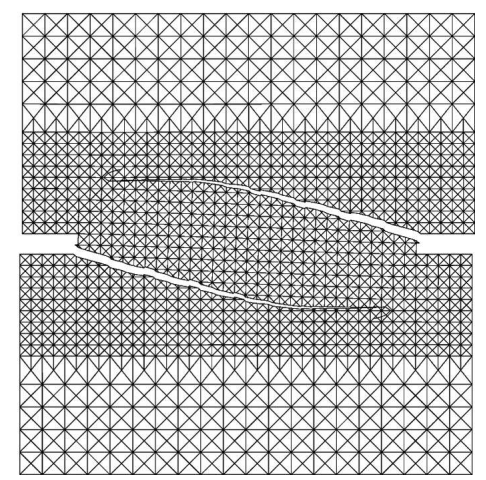

Fig. 22. Nooru-Mohamed [33] test: calculated cracks for different relative minimum distances from element vertices: a) $1 \%$, b) $2 \%$, c) $10 \%$ and d) $20 \%$ (crack model by Cox [26]). 


\section{Conclusions AND future Plans}

Some important numerical aspects of the eXtended Finite Element Method were presented. Three different benchmarks concerning cracked plan concrete specimens were numerically analysed.

Numerical simulations' results showed that the influence of the finite element formulation or integration scheme had an insignificant effect on FE results. Also a choice of a discrete constitutive law did not significantly affect the concrete response. The most important issue was the condition for the direction of crack propagation. This direction assumed as perpendicular to the direction of the maximum principal stress was wrong during bending and was overestimated during mixed tension-shear. At present, this aspect of the XFEM formulation is intensively investigated. In addition, a coupling between a continuous non-local approach and a discontinuous XFEM approach is under the implementation process. This will allow us for FE simulations of the entire fracture process in concrete.

\section{AKNOWLEDGEMENTS}

Scientific work has been carried out as a part of the Project: "Innovative resources and effective methods of safety improvement and durability of buildings and transport infrastructure in the sustainable development" financed by the European Union (POIG.01.01.02-10-106/09-01).

The FE calculations were carried out at the Academic Computer Center in Gdańsk.

\section{REFERENCES}

1. Z.P. BažAnt, J. Planas, Fracture and size effect in concrete and other quasi-brittle materials. CRC Press LLC, Boca Raton, 1998.

2. G. Lillu, J.G.M. van Mier, $3 D$ lattice type fracture model for concrete, Engineering Fracture Mechanics, 70(7-8), 927-941, 2003.

3. J. Tejchman, J. Bobiński, Continuous and discontinuous modeling of fracture in concrete using FEM. Springer, Berlin-Heidelberg (eds. W. Wu and R. I. Borja), 2012.

4. M. Ortiz, A. PANDolfi, Finite-deformation irreversible cohesive elements for three-dimensional crackpropagation analysis, International Journal for Numerical Methods in Engineering, 44(9), 1267-1282, 1999.

5. J.C. Galvez, J. Cervenka, D.A. Cendon, V. Saouma, A discrete crack approach to normal/shear cracking of concrete, Cement and Concrete Research, 32(10), 1567-1585, 2002.

6. F. Zhou, J.F. Molinari, Dynamic crack propagation with cohesive elements: a methodology to address mesh dependency, International Journal for Numerical Methods in Engineering, 59(1), 1-24, 2004.

7. T. Belytschкo, J. Fish, B.E. Englemann, A finite element method with embedded localization zones, Computer Methods in Applied Mechanics and Engineering, 70(1), 59-89, 1988.

8. M. JiRASEK, Comparative study on finite element with embedded discontinuities, Computer Methods in Applied Mechanics and Engineering, 188(1-3), 307-330, 2000. 
9. T. Belytschko, N. Möes, S. Usui, C. Parimi, Arbitrary discontinuities in finite elements, International Journal for Numerical Methods in Engineering, 50(4), 993-1013, 2001.

10. A. Simone, L.J. Sluys, The use of displacement discontinuities in a rate-dependent medium, Computer Methods in Applied Mechanics, 193(27-29), 3015-3033, 2004.

11. J. Oliver, A.E. Huespe, P.J. SAnchez, A comparative study on finite elements for capturing strong discontinuities: E-FEM vs X-FEM, Computer Methods in Applied Mechanics and Engineering, 195(37-40), 4732-4752, 2006.

12. J.M. Melenk, I. BabušKa, The partition of unity finite element method: basic theory and applications, Computer Methods in Applied Mechanics and Engineering, 139(1-4), 289-314, 1996.

13. Т. BеLYTsChко, T. BlaCK, Elastic crack growth in finite elements with minimal remeshing, International Journal for Numerical Methods in Engineering, 45(5), 601-620, 1999.

14. N. Mö̈s, T. Belytschкo, A finite element method for crack growth without remeshing, International Journal for Numerical Methods in Engineering, 46(1), 131-150, 1999.

15. C. Daux, N. Möes, J. Dolbow, N. Sukumar, T. Belytschкo, Arbitrary branched and intersecting cracks with the extended finite element method, International Journal for Numerical Methods in Engineering, 48(12), 1741-1760, 2000.

16. N. Sukumar, N. Mö̈s, T. Belytschko, B. Moran, Extended finite element method for three-dimensional crack modelling, International Journal for Numerical Methods in Engineering, 48(11), 1549-1570, 2000.

17. G.N. Wells, L.J. SluYs, A new method for modelling cohesive cracks using finite elements, International Journal for Numerical Methods in Engineering, 50(12), 2667-2682, 2001.

18. N. Möes, T. Belytschкo, Extended finite element method for cohesive crack growth, Engineering Fracture Mechanics. 69(7), 813-833, 2002.

19. G. ZI, T. BеLYTschко, New crack-tip elements for XFEM and applications to cohesive cracks, International Journal for Numerical Methods in Engineering, 57(15), 2221-2240, 2003.

20. J. Mergheim, E. Kuhl, P. Steinman, A finite element method for the computational modelling of cohesive cracks, International Journal for Numerical Methods in Engineering, 63(2), 276-289, 2005.

21. A. Hansbo, P. HansBo, A finite element method for the simulation of strong and weak discontinuities in solid mechanics, Computer Methods in Applied Mechanics and Engineering, 193(33-35), 3523-3540, 2004.

22. J.-H. Song, P.M.A. Areias, T. Belytschro, A method for dynamic crack and shear zone propagation with phantom nodes, International Journal for Numerical Methods in Engineering, 67(6), 868-893, 2006.

23. T. RabczuK, G. ZI, A. Gerstenberger, W.A. Wall, A new crack tip element for the phantom-node method with arbitrary cohesive cracks, International Journal for Numerical Methods in Engineering, 75(5), 577-599, 2008.

24. S. Mariani, U. Perego, Extended finite element method for quasi-brittle fracture, International Journal for Numerical Methods in Engineering, 58(1), 103-126, 2003.

25. J. Oliver, A.E.Huespe, E. Samaniego, E.W. Chaves, Continuum approach to the numerical simulation of material failure in concrete, International Journal for Numerical and Analytical Methods in Geomechanics, 28(7-8), 609-632, 2004.

26. J.V. Cox, An extended finite element method with analytical enrichment for cohesive crack modelling, International Journal for Numerical Methods in Engineering, 78(1), 48-83, 2009.

27. J.F. Unger, S. EcKardt, C. KonKe, Modelling of cohesive crack growth in concrete structures with the extended finite element method, Computer Methods in Applied Mechanics and Engineering, 196(41-44), 4087-4100, 2007.

28. J.L. Asferg, P.N. Poulsen, L.O. Nielsen, A consistent partly cracked XFEM element for cohesive crack growth, International Journal for Numerical Methods in Engineering. 72(4), 464-485, 2007. 
29. P. DumstorfF, Modellierung und numerische Simulation von Rissfortschritt in sproen und quasi-sproden Materialien auf Basis der Extended Finite Element Method. Ruhr-Universitat Bochum, 2006.

30. C. Le Bellego, J.F. Dube, G. Pijaudier-Cabot, B. Gerard, Calibration of nonlocal damage model from size effect tests, European Journal of Mechanics A/Solids, 22(1), 33-46, 2003.

31. A. Rodriguez-Ferran, I. Morata, A. Huerta, Numerical modelling of notched specimens, Proc. of the fifth World Congress on Computational Mechanics WCCM V, Vienna Austria, 2002.

32. L. SkARŻyński, J. Tejchman, Calculations of fracture process zones on meso-scale in notched concrete beams subjected to three-point bending, European Journal of Mechanics/A Solids, 29(4), 746-760, 2010.

33. M.B. Noonu-Mohamed, Mixed mode fracture of concrete: an experimental approach, PhD Thesis, Delft University of Technology, 1992.

Remarks on the paper should be sent to the Editorial Office no later than March 30, 2013
Received Jun 6, 2012 revised version December 4, 2012 